\title{
Short and Intermediate Outcome of Endovascular Aortic Aneurysmal Repair, A multicenteric Study
}

\author{
Mohamed Hosny Sayed ${ }^{1 *}$, Mohammed Ali Hassan ${ }^{1}$, Ahmed Samir Hosny ${ }^{1}$, Hisham Rashid ${ }^{2}$, Mohamed Hosni El Dessoki ${ }^{1}$ \\ ${ }^{1}$ Department of Vascular Surgery, Cairo University Hospitals, Cairo, Egypt; ${ }^{2}$ Department of Vascular Surgery, KCH NHS \\ Foundation Trust, Kings College, London, England
}

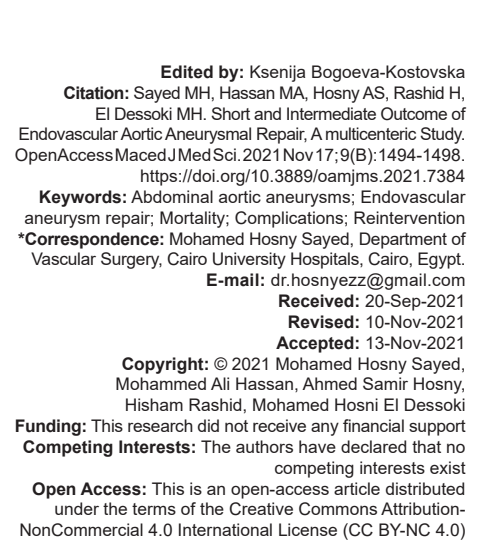

\section{Abstract}

BACKGROUND: The availability of aortic stent-grafts has permitted an obvious change in the management of abdominal aortic aneurysms (AAA). For elective cases open surgical repair has been widely superseded by the use of stent-graft. With the rapid evolution of the endovascular technology, a significant development in stent-graft techniques was achieved in conjunction with a better understanding of how to utilize stent-grafts.

METHODS: A multicenter prospective study in which 49 patients were enrolled, they underwent an elective endovascular aneurysm repair (EVAR) procedure for infrarenal AAA in two different institutions, Kasr Al-ainy School of Medicine, Cairo University and King's College Hospital in London, the selected patients were followed up for 6 months after the procedure. Data relating to demographics and pre-operative comorbidities were recorded Aneurysm morphology was reviewed by computed tomography angiography scans. Clinical data was collected through operative records and afterward through outpatient clinic follow-up sessions.

RESULTS: Overall survival was $94 \%$ over a period of 6 months calculated using KaplanMeier Survival Curve

CONCLUSIONS: Endovascular repair resulted in fewer perioperative deaths. This study provides insight into clinical parameters that can be used to stratify patients' post-EVAR surveillance and need for re-intervention and it came to the conclusion that EVAR could be considered as the standard repair for uncomplicated infrarenal AAA.

\section{Introduction}

Aneurysm formation mostly involves the infrarenal segment of the abdominal aorta. These aneurysms were responsible for approximately 13,000 deaths in 1997 [1]. For elective cases open surgical repair was widely superseded by the deployment of stent-graft through minimal surgical access with common femoral arteries [2].

Endovascular aneurysm repair (EVAR) was first undertaken by a Ukrainian surgeon Nicholas Volodos in 1987; however, it was published by Juan Carlos Parodi in 1991 in which retrograde deployment through the femoral approach of a stent-anchored with Dacron mesh graft that would isolate the aneurysmal sac and depressurize the aneurysm subsequently reducing the risk of rupture. This was the moment were the era of EVAR was born [3].

\section{Methods}

49 patients including 46 males and 3 females, who underwent an elective EVAR procedure for infrarenal abdominal aortic aneurysm (AAA), at two different institutions, Kasr Alainy School of Medicine Cairo University and King's College Hospital in London. starting from July 2016 to July 2019 , and were followed up for a period of 6 months after the procedure. Patient included in this study who has infra-renal AAA confirmed by CT angiography (CTA), with a diameter at least $5 \mathrm{~cm}$ diameter and has a compatible morphology for the manufacturer's instruction of use. While patients with supra-renal AAA or patients who need branched or fenestrated devices were excluded from the study.

Data relating to demographics and preoperative comorbidities were recorded which comprised age, gender, tobacco smoking, ischemic heart disease (IHD), obstructive lung disease, diabetes, hypertension (HTN), and the American Society of Anaesthesiologists (ASA) Physical Status Classification.

Furthermore, aneurysm morphology was reviewed by CTA scans focusing on the characters of the proximal neck in terms of diameter, length, angulation, mural thrombus, and involvement of the internal iliac arteries, also the characters of the distal landing zone in terms of iliac diameter, length, and tortuosity, the state of renal arteries and accessory renal arteries. Clinical data was collected through operative records and afterward through outpatient clinic follow-up sessions. The data of 
the patient was revised and the CTA was revised where the level of lowest renal was determined and the relevant angle was recorded. All data and measurements were confirmed on a worksheet. The available endovascular device and the required deployment accessories were re-checked. Devices used were Endurant (Medtronic), Excluder (Gore), Zenith (Cook), Incraft (Cordis), and Treovance (Bolton).

Primary outcomes of the study were assessment of mortality (30-day mortality and mortality after 6 months), also initial post-operative intensive care unit (ICU) stay, and total hospital stay were recorded. Secondary outcomes were directed for the detection of complications potentially occurring both during and after the EVAR procedure.

Patients were followed up for a period of 6 months during which the patients attended at two outpatient clinics follow-up visits (within 30 days and after 6 months). Follow-up imaging included CTA at post-operative day 2 to assess for technical success. Duplex scan was done at 1 and 6 months. Sac expansion (increase of $\geq 5 \mathrm{~mm}$ compared with pre-discharge) was assessed on each scan. Additional CTA was done in case of any abnormality detected on duplex scan.

Baseline characteristics of EVAR were compared using the $\chi^{2}$ or Fisher exact, as necessary, for discrete/categoric data. Descriptive statistics are listed as mean \pm standard deviation for continuous variables and percent (frequency) for categoric variables. Correlation between various variables was done using the Spearman rank correlation equation. Survival analysis was done using Kaplan Maier statistics calculating the mean survival time with the $95 \% \mathrm{Cl}$ and the corresponding survival graphs. Two-sided $p<0.05$ was considered statistically significant. All statistical calculations were done using computer program IBM Statistical Package for the Social Science; IBM Corp, Armonk, NY, USA) release 22 for Microsoft Windows.

\section{Results}

The mean age for the patients included in this study is $71.14 \pm 10.14$ years. The study included $46(93.9 \%)$ males. Smoking is the most common risk factor incorporated in this population (63.3\%). 59.2\% were ASA grade 3.

The mean values of the AAA maximum diameter, proximal neck diameter, and proximal neck length in the study population were $66.4 \pm 16.5 \mathrm{~mm}, 24.3 \pm 3.4 \mathrm{~mm}$, and $23.6 \pm 6.03 \mathrm{~mm}$, respectively. Percutaneous access was done in $25(51 \%)$ patients. Zenith (Cook) was the most used device $(38.7 \%)$ followed by Endurant (Medtronic) (24.8\%), Incraft (Cordis) $(20.4 \%)$, Excluder (Gore) $(8.1 \%)$ and Treovance (Bolton) $(8.1 \%)$ (Figure 1).

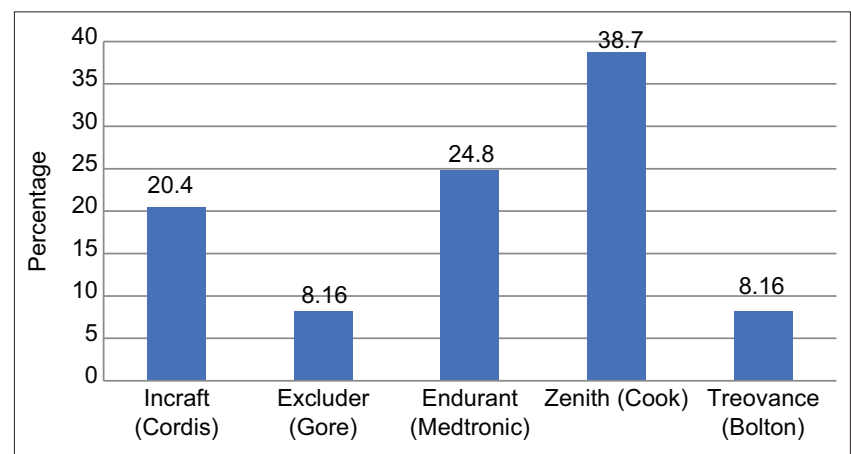

Figure 1: Pie chart showing percentages of each endovascular graft used

\section{Primary outcome measures}

Regarding mortality, the overall survival was $94 \%$ with a mortality of $6 \%$ over a period of 6 months (Figure 2). There was one case of mortality observed within the $1^{\text {st }} 30$ days due to acute myocardial infarction (MI) in ICU after 11 days. Patient was an 86-yearold man with a medical history of chronic obstructive pulmonary disease and HTN (ASA grade 3). The initial $48 \mathrm{~h}$ post-operative CT angiogram showed satisfactory appearances, however he developed on the following day, prior to discharge from ICU, chest pain and electrocardiogram changes suggestive of anterior $\mathrm{MI}$ associated with fast atrial fibrillation.

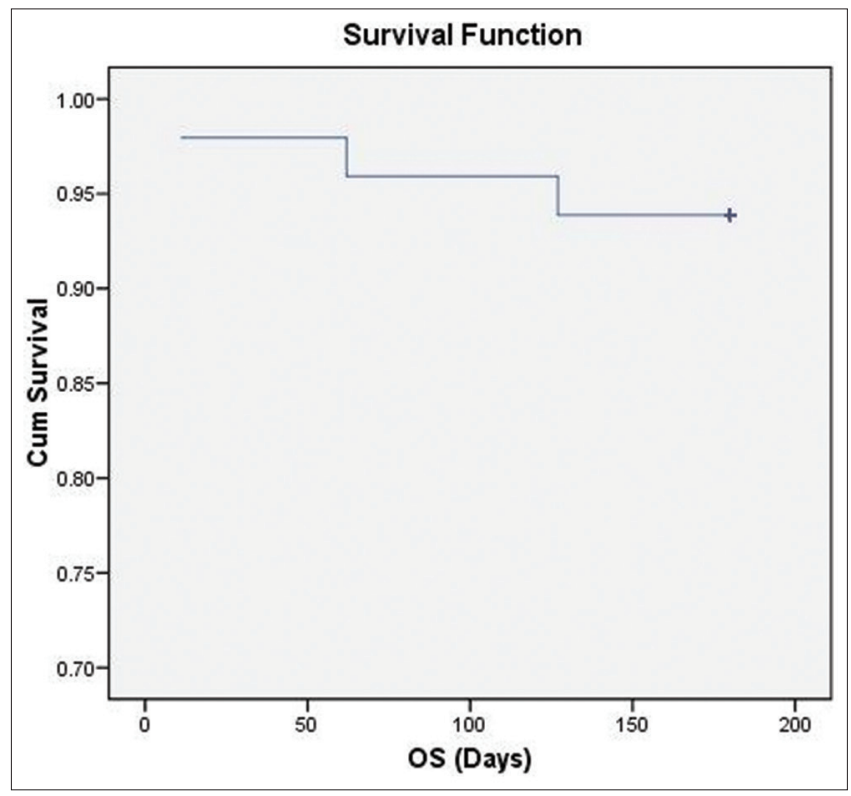

Figure 2: Survival curve over 6 months period for the study population

Two further cases of mortality were recorded within the following 6 months, after 3 months, and attributed to a cardiac event. The patient was a 66 years old female patient, ASA grade 3, with profound past medical history of significant IHD (Left ventricular impairment and aortic stenosis). She developed massive $\mathrm{Ml}$ resulting in heart failure (HF) and acute pulmonary edema and developed later hospital-acquired pneumonia, cardiac tamponade, and ventricular tachycardia cardiac arrest. The other case 
of mortality was after 4 months due to unknown cause (died at home).

Regarding ICU and total hospital stay, the average post-operative ICU stay was 1.7 days while the average total hospital stay was 7 days. There were two cases reported with lengthy ICU and hospital stay. One patient developed acute kidney injury requiring repeated sessions of hemofiltration. While the other one, he had an ICU stay of 25 days and total hospital stay of 62 days due to massive MI, pulmonary edema, hospital-acquired pnuemonia, and decompensated HF which eventually caused his death.

There was a positive correlation found between the AAA maximum diameter and the length of the ICU stay with a significant $p=0.012$ (Table 1 ).

Table 1: Correlation between AAA maximum diameter with mortality, total hospital stay, and ICU stay

\begin{tabular}{lll}
\hline AAA max diameter $(\mathrm{mm})$ & & \\
\hline Mortality & Total No. & 49 \\
& Correlation coefficient & -0.039 \\
Total hospital Stay & p-value & 0.789 \\
& Total No. & 49 \\
& Correlation coefficient & -0.171 \\
ICU Stay & p-value & 0.239 \\
& Total No & 49 \\
& Correlation coefficient & 0.356 \\
ICU: Intensive care unit. & p-value & 0.012 \\
\hline
\end{tabular}

\section{Secondary EVAR end points}

Regarding access Related Complications, there were 2 cases (4\%) complicated with common femoral artery (CFA) pseudoaneurysm, one was a complicated percutaneous access case and the other was a complicated surgical cut-down. Open repair for both cases was done with smooth outcome. Furthermore, one reported case of brachial artery pseudoaneurysm, that was repaired surgically. Perioperatively, there were 4 (8\%) Type I endoleak detected at the completion angiogram, three cases required proximal cuff extension while in the last one there was a low flow minimal endoleak and was managed conservatively, and follow-up imaging was satisfactory. There were 2 (4\%) Type II endoleak which were managed conservatively and follow-up imaging after 6 months showed no significant sac expansion. There were 2 (4\%) Type III endoleak, one (Endurant Medtronic) was detected at the completion angiogram where endolining with a long limb extension was used while the other one was detected on the $48 \mathrm{~h}$ postoperative CT and was managed later by endolining with Zenith Cook limb extension with satisfactory completion angiogram.

After 1 month, there were 2 (4\%) Type I endoleak detected on follow-up imaging. One case no re-intervention was done due to poor general condition of the patient due to acute $\mathrm{Ml}$ and he died later mainly from his cardiac event. As for the other case, small quantity of contrast was observed at the upper part of the sac on the follow-up CTA which was highly suspicious of Type I endoleak. Digital subtraction angiography was done and multiple runs in different angles showed no evidence of endoleak. There were 6 (12\%) Type II endoleak (including the previously detected two cases) which were also managed conservatively where follow-up imaging afterward showed no significant sac expansion. There was $1(2 \%)$ Type III endoleak which was managed by endolining.

After 6 months, no further new endoleak was observed at this point. All previous 6 Type II endoleak cases remained stable with no significant expansion, so they continued to be under conservative management.

There were two cases of contrast nephropathy in the immediate post-operative period, one of them required hemofiltration. No renal artery occlusion was reported. There was one case of symptomatic graftlimb occlusion with acute acute lymphoblastic leukemia pain detected after 1 month (Endurant Medtronic device) where a femoro-femoral cross over bypass was done. No reported cases of neither Bowel ischemic complications nor infection of the stent-graft or the excluded aneurysmal sac.

\section{Re-admission and re- intervention}

During the $1^{\text {st }} 30$ days, there were five cases of re-admission, four cases for management for early complications, 2 CFA pseudoaneurysm repair, a Brachial artery Pseudoaneurysm repair, Endolining for Type III endoleak and 1 case readmitted for encephalitis. After 1 month, there was 1 case $(2 \%)$ of re-admission for an occluded iliac limb (Endurant Medtronic device) to which femoro-femoral cross-over bypass was done. No re-admission cases were recorded after 6 months.

\section{Discussion}

Over the past few decades, the number of patients that was treated by EVAR has shown a great increase in their number. It started as devices that was constructed in the operating theatre then it evolved into "off-the-shelf" systems which can treat a range of patients [2]. EVAR has become the widely preferred option to treat AAA due to its fewer complication compared to open repair [3].

The European Collaborators on Stent-Graft Techniques for AAA Repair (EUROSTAR) documented $3 \%$ of 30 -day mortality. Using the $5.5 \mathrm{~cm}$ diameter of the aneurysmal sac as cutting parameter, those with AAA $>5.5 \mathrm{~cm}$ had higher mortality in comparison to patients with smaller sac diameter (4.4\% vs. $1.4 \%, p=0.002)$ [4].

The primary conclusion of the Dutch Randomised Endovascular Aneurysm Management (DREAM) trial was that EVAR had favorable early 
postoperative outcomes, also revealed less early mortality $(1.2 \%)$ and other associated systemic complications. As a final result, the cumulative survival at 2 years was $89.7 \%$ for the EVAR group (not significant) [5].

EVAR 1 trial, shown $1.7 \%$ of 30 -day mortality within the EVAR group. Generally, EVAR 1 trial highlighted the advantage of EVAR within the first 30 days but further delineated similar findings to DREAM in terms of long-term mortality data [6].

The Open Surgery Versus Endovascular Repair of Abdominal Aortic Aneurysm (OVER) trial revealed that 30-day mortality was lower in the EVAR group $(0.5 \%)$. The OVER trialists came to the conclusion that both EVAR and open repair resulted in similar longterm survival. But the perioperative mortality was lower in the EVAR group, however late rupture remained as a major concern for the EVAR group [7].

Yalcin and Tiryakioglu in 2019 had published a retrospective study comprising 85 AAA patients who underwent EVAR and followed up over an average period of 36 months, mortality was observed to be $3.5 \%$ (3 mortality cases reported). All of which was during the immediate post-operative period. There was no statistically significant difference in the mortality and reintervention rates between the age groups [8], [9].

In terms of secondary intervention, in our study, the overall re-intervention rate over the 6 months period was $4 \%$ (2 patients). In the study of Yalcin and Tiryakioglu in 2019, the secondary intervention rate was $7 \%$ (six patients); in the 36-month follow-up, reintervention was performed in six patients [8].

In the EUROSTAR series, secondary intervention was common and occurred more frequently in those with aneurysms larger than $5.5 \mathrm{~cm}$ with an annual incidence of 11.6 cases/100 patient-years [4]. In the DREAM trial, the secondary re-intervention rate in the EVAR group was $29.6 \%$ [5]. In the EVAR-1 trial, reintervention in the EVAR group was $5.1 \%$. Interestingly, the majority of these reinterventions happened within 6 months following the index procedure [6] Secondary interventions in the OVER trial were $13.7 \%$ in the EVAR group [7].

Regarding the observed complications, in our study, the total endoleak rate was about $20.4 \%$ in within 30 -day period and $18.4 \%$ after 6 months with a majority of Type II endoleak while the graft occlusion percentage was only $2 \%$. There was no statistical significance found between the endoleak and the re-intervention rate. This could be attributed to the fact most of them were Type II and did not show any significant sac expansion, therefore they were managed conservatively. In Yalcin and Tiryakioglu 2019, Endoleaks were detected in $25(29.4 \%)$ patients during the procedure with a majority for Type I endoleak in 21 cases.

In the EUROSTAR registry, total endoleak in the 30 -day interval was $16.6 \%$. The annual incidence rate $(95 \% \mathrm{Cl})$ of endoleak was 15 cases per 100 patients (13-17). Occlusion of the Endograft occurred more frequently in patients with large aneurysms with annual incidence rate (5.7 vs. 3.7 cases $/ 100$ patient-years; $p=0.01)[4]$.

In the DREAM trial, endoleak was reported in $1.2 \%$, graft occlusion was $6.4 \%$. In the OVER trial, in the endovascular repair group, there were 134 endoleaks in 110 patients $(25 \%)$, resulting in 21 secondary therapeutic procedures in 18 patients $(4.1 \%)$ which was attributed to the differences in the endovascular systems used [4]

In Han et al. in 2017, octogenarians have higher 30-day endoleak than the younger age group which was $25.8 \%$ and $21.3 \%$ respectively [10].

\section{Conclusions}

EVAR is universally accepted as a standard repair for uncomplicated infrarenal AAA. Despite rapid changes in technology, long-term survival remains similar between EVAR and open repair. Perioperative mortality is much lower with EVAR, although secondary interventions are not uncommon they could be managed by various endovascular solutions. Endovascular repair resulted in fewer perioperative deaths. Our findings support EVAR as a safe solution for managing patients with AAA and provide insight into clinical parameters that can be used to stratify patients' post-EVAR surveillance and need for reintervention.

\section{Study limitations}

This study had several limitations include the small number of patients. Furthermore, the different types of stent grafts used could affect the net results. Longer-term data are needed to fully assess the relative merits. Heterogeneity in patient enrolment should be noted.

\section{References}

1. Cronenwett JL, Johnston KW. Rutherford's Vascular Surgery E-Book. Amsterdam, Netherlands: Elsevier Health Sciences; 2014.

2. England A, Mc Williams R. Endovascular aortic aneurysm repair (EVAR). Ulster Med J. 2013;82(1):3-10. PMid:23620623

3. Rich N, Tucker LY, Okuhn S, Hua H, Hill B, Goodney P, et al. Long-term freedom from aneurysm-related mortality remains favorable after endovascular abdominal aortic aneurysm repair in a 15-year multicenter registry. J Vasc Surg. 2020;71(3):790-8. 
https://doi.org/10.1016/j.jvs.2019.05.043.

PMid:31495678

4. Propper BW, Abularrage CJ. Long-term safety and efficacy of endovascular abdominal aortic aneurysm repair. Vasc Health Risk Manag. 2013;9:135-41. https://doi.org/10.2147/vhrm. s32250

PMid:23579199

5. De Bruin JL, Baas AF, Buth J, Prinssen M, Verhoeven EL, Cuypers PW, et al. Long-term outcome of open or endovascular repair of abdominal aortic aneurysm. N Engl J Med. 2010;362(20):1881-9. https://doi.org/10.1056/ NEJMoa0909499.

PMid:20484396

6. Greenhalgh RM, The E. Comparison of endovascular aneurysm repair with open repair in patients with abdominal aortic aneurysm (EVAR trial 1), 30-day operative mortality results: Randomised controlled trial. Lancet. 2004;364(9437):843-8. https://doi.org/10.1016/S0140-6736(04)16979-1

PMid:15351191

7. Lederle FA, Freischlag JA, Kyriakides TC, Matsumura JS, Padberg FT Jr., Kohler TR, et al. Long-term comparison of endovascular and open repair of abdominal aortic aneurysm. N Engl J Med. 2012;367(21):1988-97. https://doi.org/10.1056/ nejmoa1207481

PMid:23171095

8. Yalcin M, Tiryakioglu O. Single-center study comparing short and mid-term results of EVAR in old and young populations. Braz J Cardiovasc Surg. 2019;34(3):279-84. https://doi. org/10.21470/1678-9741-2018-0021

PMid:31310465

9. Deery SE, Ergul EA, Schermerhorn ML, Siracuse JJ, Schanzer A, Goodney PP, et al. Aneurysm sac expansion is independently associated with late mortality in patients treated with endovascular aneurysm repair. J Vasc Surg. 2018;67(1):157-64. https://doi.org/10.1016/j.jvs.2017.06.075 PMid:28865980

10. Han Y, Zhang S, Zhang J, Ji C, Eckstein HH. Outcomes of endovascular abdominal aortic aneurysm repair in octogenarians: Meta-analysis and systematic review. Eur J Vasc Endovasc Surg. 2017;54(4):454-63. https://doi.org/10.1016/j. ejvs.2017.06.027

PMid:28822680 